\title{
Preparação de sistemas nanoparticulados para a solubilização e incorporação de ativos hidrofóbicos ${ }^{1}$
}

\author{
Preparation of nanoparticulate systems for solubilization and \\ incorporation of hydrophobic actives \\ Bruna Alves de Oliveira ${ }^{2}$ \\ Kézia Cristine Barbosa Ferreira ${ }^{3}$ \\ Nádia Rezende Barbosa Raposo 4 \\ Frederico Pittella ${ }^{5}$
}

DOI: https://doi.org/10.34019/2179-3700.2018.v18.29870

\begin{abstract}
Resumo
Este estudo propõe preparar sistemas nanocarreadores para solubilização e incorporação de ativos hidrofóbicos e realizar sua caracterização físico-química. Um sistema nanoparticulado composto por lipídios de origem natural e copolímeros em bloco foi designado para a incorporação de dois ativos hidrofóbicos, constituindo duas formulações nanoestruturadas diferentes. Após a determinação do ponto de fusão e perfil cromatográfico da manteiga natural empregada, as formulações foram preparadas por fusão-emulsificação, sendo aquecidas e homogeneizadas em aparelhos apropriados. Em seguida, as formulações foram caracterizadas por Espalhamento Dinâmico de Luz. As formulações 1 e 2 obtiveram um tamanho de 124,8 \pm $0,31 \mathrm{~nm}$ e $271,9 \pm 0,28 \mathrm{~nm}$; índice de polidispersão $0,249 \pm 0,09$ e $0,255 \pm 0,07$; potencial zeta $15,3 \pm 0,78 \mathrm{mV}$ e $-11,8 \pm 0,92 \mathrm{mV}$, respectivamente. Imagens obtidas por Microscopia Eletrônica de Transmissão indicam um formato esférico das partículas com média de tamanho de aproximadamente $200 \mathrm{~nm}$. O método elaborado revelou-se eficaz para a solubilização dos ativos hidrofóbicos testados: houve formação de nanoestruturas incorporando os ativos nas condições avaliadas, com características fisico-químicas apropriadas para aplicação farmacêutica.
\end{abstract}

Palavras-chave: Nanotecnologia. Lipossolubilidade. Carreadores lipídicos nanoestruturados.

\begin{abstract}
This study proposes the preparation of nanoparticule systems for solubilization and incorporation of hydrophobic assets and to carry out its physicochemical characterization. A nanoparticulate system composed of naturally occurring lipids and block copolymers was designed to incorporate two hydrophobic actives separately, constituting two different nanostructured formulations. After determination of the melting point and chromatographic profile of the natural butter employed, the formulations were prepared by melt-emulsification, being heated and homogenized in suitable

\footnotetext{
${ }^{1}$ Trabalho premiado no Seminário de Iniciação Científica da UFJF em 2015.

${ }^{2}$ Bolsista do Programa de apoio ao recém-doutor (Enxoval).

${ }^{3}$ Colaboradora no projeto - Departamento de Ciências Farmacêuticas/Faculdade de Farmácia, Universidade Federal de Juiz de Fora (UFJF).

${ }^{4}$ Colaboradora no projeto - Departamento de Ciências Farmacêuticas/Faculdade de Farmácia, Universidade Federal de Juiz de Fora (UFJF).

${ }^{5}$ Professor orientador - Departamento de Ciências Farmacêuticas/Faculdade de Farmácia, Universidade Federal de Juiz de Fora (UFJF), e-mail: frederico.pittella@ufjf.edu.br
} 
apparatus. Then the formulations were characterized by Dynamic Light Scattering. Formulations 1 and 2 presented a size of $124.8 \pm 0.31 \mathrm{~nm}$ and $271.9 \pm 0.28 \mathrm{~nm}$; polydispersity index $0.249 \pm 0.09$ and $0.255 \pm 0.07$; zeta potential $-15.3 \pm 0.78 \mathrm{mV}$ and $-11.8 \pm 0.92 \mathrm{mV}$, respectively. Images obtained by Transmission Electron Microscopy indicate a spherical shape of the particles with an average size of approximately $200 \mathrm{~nm}$. The elaborated method proved to be effective for the solubilization of hydrophobic assets tested: nanostructures were formed incorporating the assets under the evaluated conditions, with physico-chemical characteristics appropriate for pharmaceutical application.

Keywords: Nanotechnology. Hydrophobicity. Nanostructured lipid carriers.

\section{INTRODUÇÃO}

A descoberta e o desenvolvimento de um novo fármaco requer relevante investimento financeiro, uma vez que a cada 20.000 moléculas promissoras que alçam os estudos pré-clínicos, apenas uma chega ao mercado (BARREIRO; FRAGA, 2015; BARREIRO; PINTO, 2013). Ademais, para certas patologias, como o câncer, os fármacos apresentam grau de cura dito como "limitado", em razão das repetidas doses clínicas necessárias, para suprimir a perda da distribuição dos ativos no organismo e o avanço da doença. Tal fato resulta muitas vezes em efeitos adversos e em resistência ao tratamento (GASSMANN; REEPMEYER; ZEDTWITZ, 2008; SATO et al., 2011; DEMEURE et al., 2012).

Mesmo com o aumento considerável de moléculas ativas potenciais - por mérito da química combinatória, triagem de alto rendimento, biologia e genômica - cerca de $40 \%$ não avançam nas pesquisas, pois apresentam solubilidade reduzida nos líquidos do organismo dificultando o acesso à superfície das células e consequente absorção, o que explica a baixa biodisponibilidade (KUMAR et al., 2011; LOH; SAMANTA; HENG, 2015). Entretanto, a baixa solubilidade em água ou lipossolubilidade é crucial na transposição das moléculas pelas barreiras biológicas, ou seja, no processo de absorção. Desse modo, o coeficiente de partição O/A (grandeza que mede a lipossolubilidade) não pode ser alto a ponto de dificultar a dissolução dos fármacos no líquido gastrintestinal e na circulação sanguínea, pois influencia negativamente na biodisponibilidade e distribuição do fármaco respectivamente, mas precisa ser suficiente para permitir ao fármaco transpor membranas biológicas (BARREIRO; FRAGA, 2015; JANEIRO, 2011; SINHA; MÜLLER; MÖSCHWITZER, 2013).

Assim, apenas a descoberta de novos fármacos não garante a eficácia do tratamento de doenças, fazendo-se necessária a adoção de novas estratégias 
terapêuticas. Nos últimos anos o interesse nos sistemas nanoparticulados é crescente, pois estes são potenciais sistemas de entrega de fármacos. Além dissosão promissores para uma ação terapêutica mais eficiente, melhor biodistribuição e auxílio na transposição de barreiras biológicas (CHARCOSSET; EL-HARATI; FESSI, 2005; DEMEURE et al., 2012).

Os sistemas de entrega de fármacos são capazes de alterar as propriedades físicoquímicas da substância ativa e/ou interagir com estruturas biológicas de modo que superem as limitações das formas convencionais. Além da melhoria na solubilidade, estabilidade e passagem por múltiplas barreiras biológicas (KHADKA et al., 2014; ALKASSAS; BANSAL; SHAW, 2017), é possível combinar fatores como, mascaramento de características desagradáveis, favorecer o direcionamento do fármaco, modular sua liberação, absorção e distribuição, redução das doses, aumento da eficácia e aumento da compatibilidade em formulação (MERISKO-LIVERSIDGE; LIVERSIDGE, 2011).

Dentre as opções de encapsulamento de ativos com baixa solubilidade, as Nanopartículas Lipídicas Sólidas (NLS) são constituidas por lipídios sólidos à temperatura ambiente e estabilizadas normalmente por tensoativos. Foram desenvolvidas na década 1990, por Müller e colaboradores (1995) ao patentearem um método de produção de NLS por homogeneização à alta pressão. Além disso, não necessitam de solventes orgânicos no preparo (MÜLLER et al., 1995; MEHNERT; MÄDER, 2001; CHARCOSSET; ELHARATI; FESSI, 2005). Os lipídios empregados na preparação de NLS podem ser de elevada pureza como a triestearina ou uma mistura de glicerídeos ou ceras. A solidez característica dos lipídios confere menor mobilidade aos ativos neles incorporados podendo sustentar ou até controlar a velocidade de liberação destas substâncias, além de aumentar a biodisponibilidade de fármacos e reduzir a variabilidade farmacocinética de fármacos lipofílicos. O tamanho reduzido variando de 50 a 1000nm, permite que as NLS sejam empregadas em diferentes vias de administração de medicamentos, como: oral, parenteral e cutânea (SILVA et al., 2011;SOUZA et al., 2011).

O objetivo deste trabalho foi preparar nanopartículas lipídicas sólidas utilizando polímeros para estabilização, que sejam capazes de solubilizar ativos hidrofóbicos e garantir sua incorporação, bem como realizar sua caracterização físico-química. 


\section{METODOLOGIA}

Foram preparadas duas formulações de NLS com manteiga natural de amêndoa de Astrocaryum aculeatum (nome popular: tucumã) e copolímero em bloco Pluronic F68, contendo, cada uma, um princípio ativo hidrofóbico distinto: Ativo A ( Log P 2.57) e Ativo B (Log P 2.36).

Foi utilizado $1 \mathrm{~g}$ de manteiga natural na determinação do ponto de fusão do lipídio em aparelho digital de ponto de fusão MQAPF-302 (Microquímica). O ponto de fusão foi determinado pela média da triplicata. Os constituintes graxos da manteiga natural foram determinados por Cromatografia Gasosa de Alta Resolução (CG-AR), em Cromatógrafo a Gás HP5890. As amostras foram diluídas em clorofórmio 0,5\%. A identificação dos componentes químicos foi baseada no tempo de retenção da amostra e uma mistura de $n$-alcanos de C10 - C18 (CHRISTIE; HAN, 2010).

As formulações foram obtidas por fusão-emulsificação (SOUTO et al., 2011) com adaptações. A manteiga natural foi previamente fundida a $45^{\circ} \mathrm{C}$ em banho-maria e os ativos incorporados por dissolução/dispersão. Em seguida, esta fase foi emulsificada na fase aquosa contendo o bloco de copolímero por meio de alta agitação a 15.000rpm por 10 minutos (SilentCrusher M, Heidolph, Alemanha), seguida de irradiação ultrassônica a 35\% (Vibra Cell, Sonics, EUA), originando gotículas pequenas, dispersas no meio da fase aquosa formando a NLS. A solubilização dos ativos foi observada a olho nu. A emulsão recém-preparada permaneceu à temperatura ambiente para a solidificação dos lipídios e obtenção da dispersão aquosa da NLS.

Decorridas 24 horas do preparo, foi realizada a caracterização físico-química em aparelho Zetatrac (Microtrac, EUA), que fornece o diâmetro da partícula e o Índice de Polidispersão $(\mathrm{Pdl})$, através da técnica de espectroscopia de correlação de fótons ou espalhamento dinâmico de luz (DLS), e o Potencial Zeta (PZ) através da avaliação da mobilidade eletroforética. Para caracterização as amostras foram diluídas $(1: 400 \mathrm{v} / \mathrm{v})$ em água MiliQ ${ }^{\circledR}$. Os valores foram obtidos através do cálculo da média de 5 leituras.

A morfologia das partículas foi realizada por meio da Microscopia de Transmissão (MET). As dispersões de NLS foram diluídas (1:5 v/v) em água Mili $Q^{\circledR}$ e depositadas sobre um grid de metal de cobre revestido com carbono (CF 200-Cu, 300 mesh de cobre, EMS, EUA). As partículas foram visualizadas em uma magnitude de 100.000 vezes em microscópio eletrônico (MET JEM-1011, JEOL, EUA). 


\section{RESULTADOS E DISCUSSÃO}

A solubilidade é uma das mais importantes propriedades físico-químicas na descoberta de fármacos, uma vez que a hidrofobicidade elevada pode dificultar o desenvolvimento de produtos e limitar significativamente a biodisponibilidade após administração oral. Desta forma, a solubilização de ativos hidrofóbicos em nanopartículas pode contribuir para o desenvolvimento de formulações mais adequadas e promissoras, mesmo contendo fármacos pouco solúveis em água (MERISKO-LIVERSIDGE; LIVERSIDGE, 2011).

Na construção de um sistema nanoestruturado, a caracterização dos constituintes a serem empregados é fundamental para determinar o tipo de nanocarreador a ser produzido e o seu método de preparo. A manteiga natural empregada neste estudo apresentou ponto de fusão de $33,9^{\circ} \mathrm{C}$. A constituição química da manteiga (Figura 1) contém cinco ácidos graxos: C18:1 - oleico (66,6\%), C16:0 - palmítico (21,2\%), C18:2 linoleico $(5,3 \%)$, C18:0 - esteárico $(5,1 \%)$ e C18:3 - linolênico (1,8\%). A porcentagem balanceada dos ácidos graxos saturados e insaturados encontrada pode favorecer maior porcentagem de encapsulação e modulação da liberação (GASCO, 2007).

$\mathrm{Na}$ avaliação visual a olho nu da dispersão, foi verificada boa solubilização dos ativos hidrofóbicos. A caracterização físico-química (Figura 2 e Tabela 1) apresentou a média de distribuição de tamanho dentro da escala nanométrica - 50 a 1000nm prevista para o sistema em estudo (KHERADMANDNIAet al., 2010). As formulações 1 e 2 obtiveram diâmetro hidrodinâmico de 124,8 $\pm 0,31 \mathrm{~nm}$ e $271,9 \pm 0,28 \mathrm{~nm}$; índice de polidispersão $0,249 \pm 0,09$ e $0,255 \pm 0,07$; potencial zeta $-15,3 \pm 0,78 \mathrm{mV}$ e $-11,8 \pm$ $0,92 \mathrm{mV}$, respectivamente. $\mathrm{O}$ histograma calculado em número, mostra pico de tamanho em cerca de $300 \mathrm{~nm}$ para ambas as formulações (Figura 2B e 2D). Digno de nota, os histogramas de intensidade (Figura 2) possuem uma única distribuição de tamanho, o que reflete positivamente no valor do índice de polidispersão $(\mathrm{Pdl})$. O Pdl está relacionado à variância de distribuição de tamanho, e os valores encontrados indicam amostras com valores ideais para a aplicação farmacêutica (SHAW, 2012).

A fim de confirmar os dados fornecidos na análise por DLS, examinou-se a morfologia das NLS por MET (Figura 3). As imagens revelam nanopartículas em formato arredondado, com distribuição homogênea e a média de tamanho próxima a 300nm. Tais resultados estão de acordo com os histogramas apresentados na Figura 2B e 2D, obtidas 
por

DLS, mostrando que ambas técnicas atuam de forma complementar na caracterização das nanopartículas.

Figura 1 -Perfil cromatográfico da manteiga natural. Foram caracterizados cinco ácidos graxos: C18:1 - oleico (66,6\%), C16:0 - palmítico (21,2\%), C18:2 - linoleico (5,3\%), C18:0 - esteárico $(5,1 \%)$ e C18:3 - linolênico $(1,8 \%)$. As análises por CG-AR foram realizadas em Cromatógrafo a Gás HP5890, equipado com detector de ionizador de chamas, com coluna capilar HP-INNOWax $15 \mathrm{~m}$ de comprimento $\times 0,25 \mathrm{~mm}$ de diâmetro interno. $\mathrm{O}$ hidrogênio foi utilizado como gás de arraste, com um fluxo constante de $2 \mathrm{~mL} \mathrm{~min}^{-1}$, temperatura inicial do forno de $80^{\circ} \mathrm{C}$ (1 minuto), e rampa de $7^{\circ} \mathrm{C} \mathrm{min}^{-1}$ até temperatura final de $240^{\circ} \mathrm{C}$. $\mathrm{O}$ injetor foi utilizado no modo split 150 a $250^{\circ} \mathrm{C}$ e o detector na mesma temperatura. As amostras foram diluídas em clorofórmio $0,5 \%$ e o volume de injeção foi de $1 \mu \mathrm{L}$. A identificação dos componentes químicos foi baseada no tempo de retenção da amostra e uma mistura de $n$-alcanos de C10 - C18.

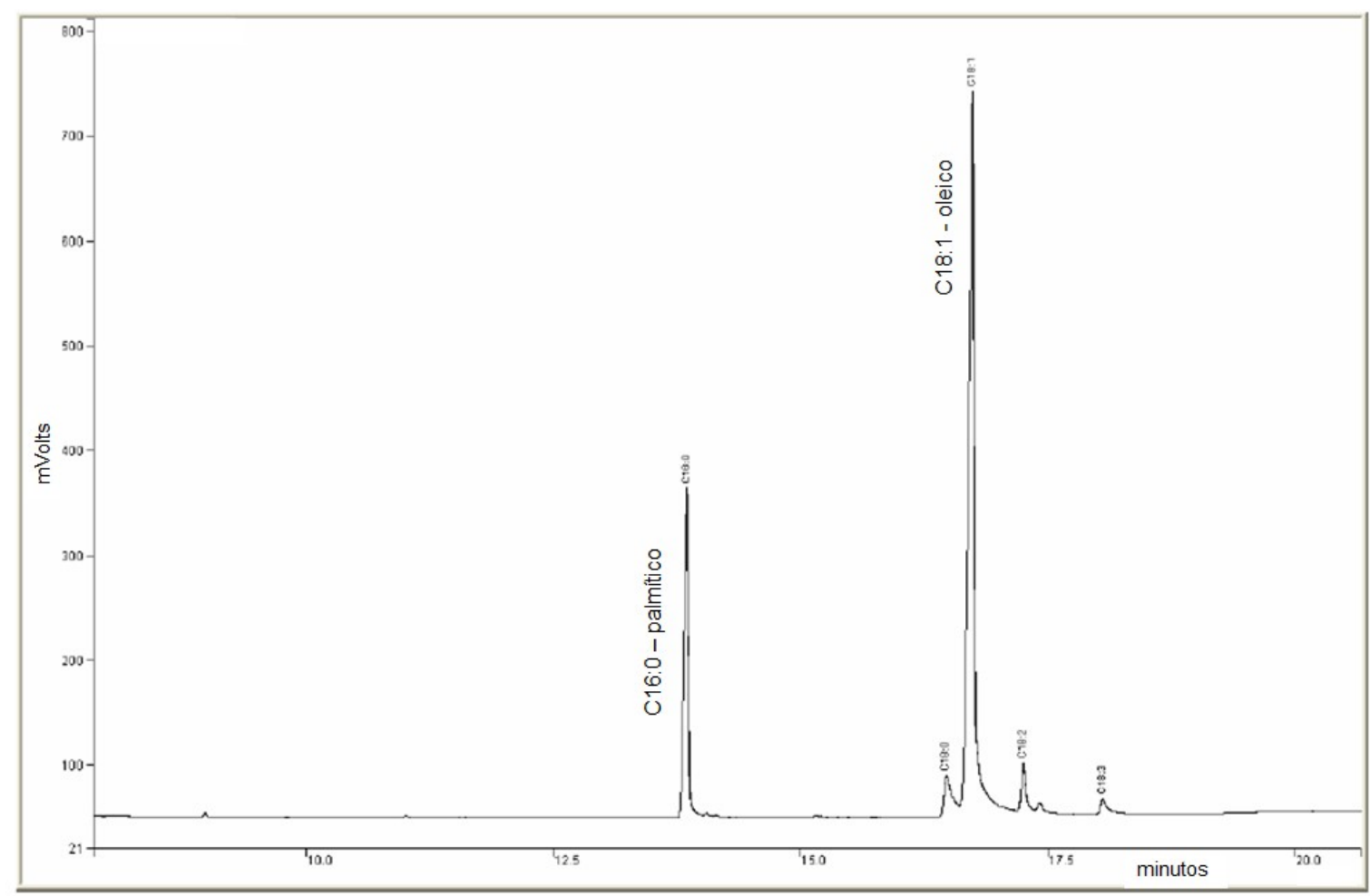

Fonte: elaboração própria. 
Tabela 1 -Caracterização físico química das formulações obtidas. As dispersões foram diluídas (1:400) em água MiliQ ${ }^{\circledR}$ e as leituras realizadas em aparelho Zetatrac (Microtrac, EUA). Os valores obtidos referem-se à média de 5 leituras. Diâmetro hidrodinâmico, Pdl (Índice de Polidispersão), DP (desvio padrão).

\begin{tabular}{cccc}
\hline Formulação & $\begin{array}{c}\text { Diâmetro } \\
\text { hidrodinâmico } \\
(\mathbf{n m}) \pm \mathbf{D P}\end{array}$ & $\begin{array}{c}\text { Índice de polidispersão } \\
\mathbf{( P d l}) \pm \mathbf{D P}\end{array}$ & $\begin{array}{c}\text { Potencial zeta } \\
(\mathbf{m V}) \pm \text { DP }\end{array}$ \\
\hline $\mathbf{1}$ & $124,8 \pm 0,31$ & $0,249 \pm 0,09$ & $-15,3 \pm 0,78$ \\
$\mathbf{2}$ & $271,9 \pm 0,28$ & $0,255 \pm 0,07$ & $-11,8 \pm 0,92$ \\
\hline
\end{tabular}

Fonte: elaboração própria

Figura 2 - Histogramas de distribuição de tamanho obtidos pela técnica de Espalhamento Dinâmico de Luz. Histogramas de intensidade do tamanho de partícula da formulação 1 (A) e formulação 2 (C), histogramas de número do tamanho de partícula da formulação 1 (B) e formulação 2 (D).

A

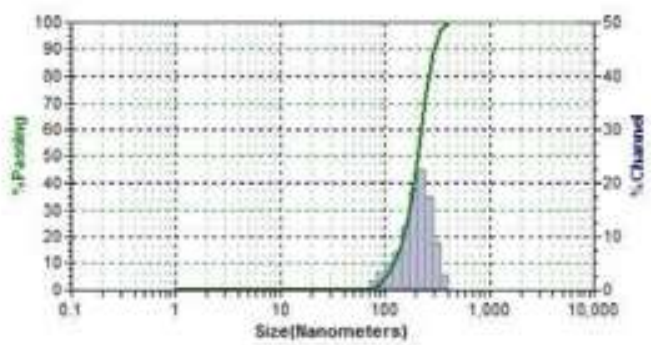

C

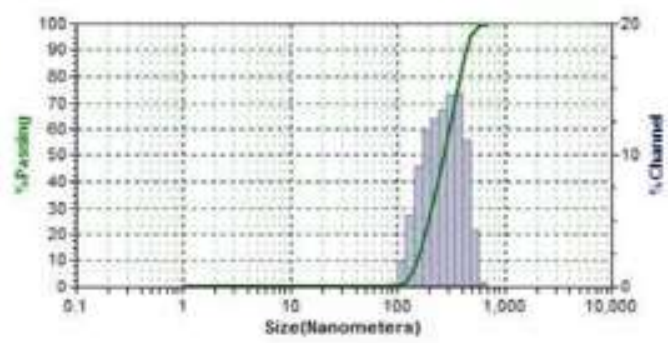

B

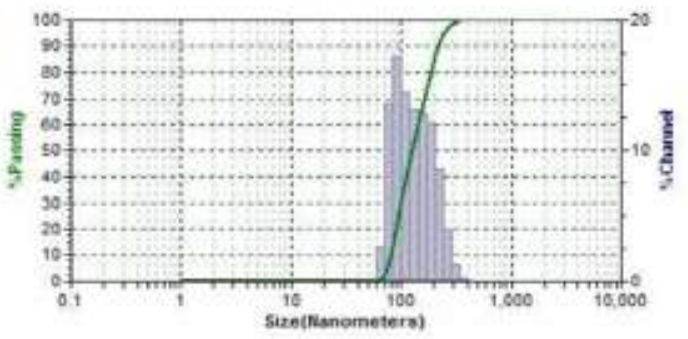

D

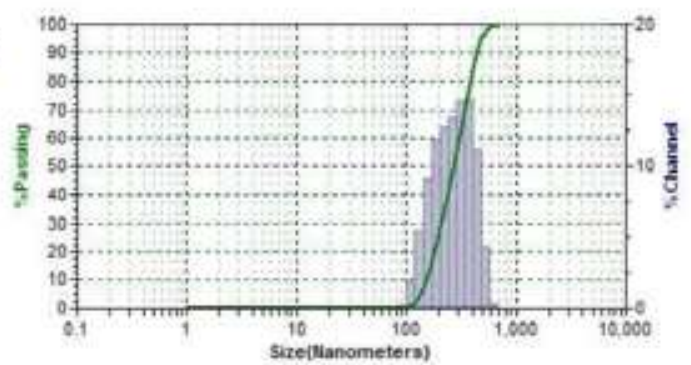

Fonte: elaboração própria. 
Figura 3 - Eletromicrografia de transmissão dos carreadores lipídicos nanoestruturados desenvolvidos. Previamente à análise por MET, as dispersões foram diluídas (1:5) em água ultrapura e depositadas sobre um grid de metal de cobre revestido com carbono (CF 200-Cu, 300 mesh de cobre, EMS, EUA). A amostra foi seca em fluxo de nitrogênio e deixada à temperatura ambiente por $12 \mathrm{~h}$. As nanopartículas foram visualizadas em uma magnitude de 100.000 vezes em um microscópio eletrônico (MET JEM-1011, JEOL, EUA). Nanopartículas da formulação 1 (A) e Nanopartículas da formulação 2 (B).

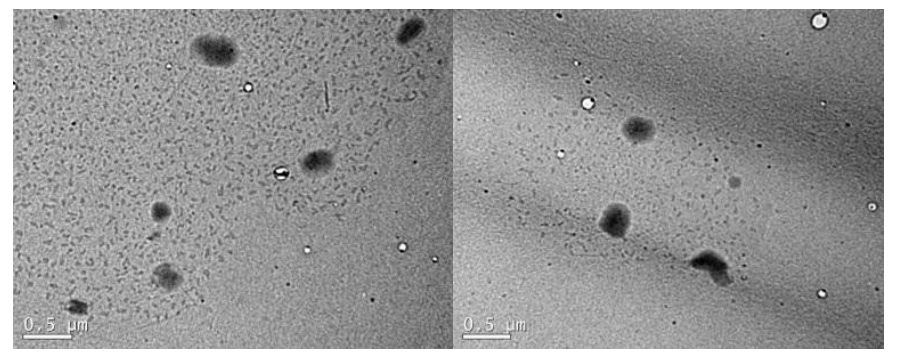

Fonte: elaboração própria.

\section{CONCLUSÃO}

No presente estudo, foi possível o desenvolvimento de sistemas nanoestruturados baseados em lipídios e estabilizados por polímero. Nas condições experimentais testadas, o sistema garantiu a solubilização dos ativos hidrofóbicos na NLS, sendo, portanto, um promissor carreador para fármacos de baixa solubilidade.

\section{AGRADECIMENTOS}

Os autores agradecem à UFJF, aos parceiros do Grupo de Estudos de Interações entre Micro e Macromoléculas (GEIMM), ao apoio financeiro da Fundação de Amparo à Pesquisa do Estado de Minas Gerais (FAPEMIG), da Coordenação de Aperfeiçoamento de Pessoal de Nível Superior (CAPES) e do Conselho Nacional de Desenvolvimento Científico e Tecnológico (CNPq).

\section{REFERÊNCIAS}

AL-KASSAS, R.; BANSAL, M.; SHAW, J. Nanosizing techniques for improving bioavailability of drugs. Journal of Controlled Release, v. 260, p. 202-212, 2017.

BARREIRO, E. J.; FRAGA, C. A. M.Química Medicinal: As bases moleculares da ação dos fármacos. 3. ed. Porto Alegre: Artmed, 2015. 
BARREIRO, E. J.; PINTO, A. C. Oportunidades e Desafios para a Inovação em Fármacos: Agora ou Nunca! Revista Virtual de Química, v. 5, n. 6, p. 1059-1074, 2013.

CHRISTIE, W. W.; HAN, X. Lipid Analysis: Isolation, separation identification and lipidomic analysis. 4. ed. Saint Louis: The Oily Press, 2010.

CHARCOSSET, C.; EL-HARATI, A.; FESSI, H. Preparation of solid lipid nanoparticles using a membrane contactor. Journal of Controlled Release, v. 108, n. 1, p.112-120, 2005.

DEMEURE, M. J.; STEPHAN, E.; SINARI, S.; MOUNT, D.; GATELY, S.; GONZALES, P.; HOSTETTER, G.; KOMOROWSKI, R.; KIEFER, J.; GRANT, C. S.; HAN, H.; VON HOFF, D. D.; BUSSEY, K. J. Preclinical Investigation of Nanoparticle Albumin-Bound Paclitaxel as a Potential Treatment for Adrenocortical Cancer. Annals of Surgery, v. 255, n. 1, p.140146, 2012.

GASCO, M. R. Lipid Nanoparticles: Perspectives and challenges. Advanced Drug Delivery Reviews,v.59, n.6, p.377-378, 2007.

GASSMANN, O.; REEPMEYER, G.; ZEDTWITZ, M. V.Leading Pharmaceutical Innovation: Trends and Drivers for Growth in the Pharmaceutical Industry.2.ed. Springer, 2008.

KHADKA, P.; RO, J.; KIM, H.; KIM, I.; KIM, J. T.; KIM, H.; CHO, J. M.; YUN, G.; LEE, J. Pharmaceutical particle technologies: An approach to improve drug solubility, dissolution and bioavailability. Asian Journal of Pharmaceutical Sciences, v. 9, n. 6, p. 304-316, 2014.

KHERADMANDNIA, S.; VASHEGHANI-FARAHANI, E.; NOSRATI, M.; ATYABI, F. Preparation and characterization of ketoprofen-loaded solid lipid nanopaticles made form beeswax and carnauba wax. Nanomedicine: Nanotechnology, Biology and Medicine, v. 6, n. 6, p.753-759, 2010.

KUMAR, A.; SAHOO, S. K.; PADHEE, K.; KOCHAR, P. P. S.; SATAPATHY, A.; PATHAK, N. Review on solubility enhancement techniques for hydrophobic drugs. PharmacieGlobale: International Journal of Comprehensive Pharmacy, v. 2, n. 3, 2011.

LOH, Z. H.; SAMANTA, A. K.; HENG, P. W. S. Overview of milling techniques for improving the solubility of poorly water-soluble drugs. Asian Journal of Pharmaceutical Sciences, v.10, n. 4, p. 255-274, 2015.

MEHNERT, W.; MÄDER, K. Solid Lipid Nanoparticles: Production, characterization and applications. Advanced Drug Delivery Reviews, v. 47, n. 2-3, p. 165-196, 2001.

MERISKO-LIVERSIDGE, E.; LIVERSIDGE, G. G. Nanosizing for oral and parenteral drug delivery: a perspective on formulating poorly-water soluble compounds using wet media milling technology. Advanced Drug Delivery Reviews, v. 63, n. 6, p. 427-440, 2011.

MÜLLER, H. R.; MEHNERT, W.; LUCKS, J. S.; SCHWARZ, C.; MÜHLEN, A. Z.; WEYHERS, H.; FREITAS, C.; RÜHL, D.; MULLER, R. H.; MUHLEN, A. Z.; MEYHERS, H.; 
RUHL, D.; MUHLEN, A. Solid lipid nanoparticles (SLN) - An alternative colloidal carrier system for controlled drug delivery. European Journal of Pharmaceutics and Biopharmaceutics, v. 41, n. 1, p. 62-69, 1995.

SATO, T.; NEILSON, L. M.; PECK, A. R.; LIU, C.; TRAN, T.H.; WITKIEWICZ, A.; HYSLOP, T.; NEVALAINEN, M. T.; SAUTER, G.; RUI, H. Signal transducer and activator of transcription-3 and breast cancer prognosis. American Journal of Cancer Research, v.1, n. 3, p. 347-355, 2011.

SILVA, A. C.; GONZÁLEZ-MIRA, E.; GARCÍA, M. L.; EGEA, M. A.; FONSECA, J.; SILVA, R.; SANTOS, D.; SOUTO, E. B.; FERREIRA, D. Preparation, characterization and biocompatibility studies on risperidone-loaded solid lipid nanoparticles (SLN): high pressure homogenization versus ultrasound. Colloids and Surfaces B: Biointerfaces, v. 86, n. 1, p. 158-165, 2011.

SINHA, B.; MÜLLER, R. H.; MÖSCHWITZER, J. P. Bottom-up approaches for preparing drug nanocrystals: Formulations and factors affecting particle size. International Journal of Pharmaceutics, v. 453, n. 1, p. 126-141, 2013.

SOUZA, L. G.; SILVA, E. J.; MARTINS, A. L.; MOTA, M. F.; BRAGA, R. C.; LIMA, E. M.; VALADARES, M. C.; TAVEIRA, S. F.; MARRETO, R. N. Development of topotecan loaded lipid nanoparticles for chemical stabilization and prolonged release. European Journal of Pharmaceutics and Biopharmaceutics, v.79, n. 1, p. 189-196, 2011.

SOUTO, E. B.; SEVERINO, P.; SANTANA, M. H. A.; PINHO, S. C. Nanopartículas de lipídios sólidos: métodos clássicos de produção laboratorial. Química Nova, v. 34, n. 10, p.1762-1769, 2011.

SHAW, R. Dynamic Light Scattering Training - Achieving reliable nano particle sizing, Malvern-Zetasizer, 2012. Disponível em: <www.atascientific.com.au>. 